\title{
Money without collaboration won't bring cures
}

\author{
Todd B Sherer
}

It's up to stakeholders at every stage of therapeutic development-industry and academic researchers, policymakers, patient foundations and even patients themselves - to embrace the power of collaboration. Only then will we enable translational research and push much-needed treatments to the clinic faster.

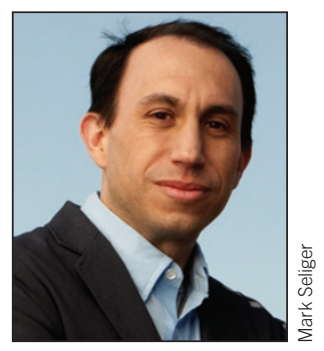

Last year, I attended the inaugural meeting of the US National Center for Advancing Translational Sciences (NCATS) advisory council, of which I am a charter member. It's an honor to serve on this council as it initiates planning around the center's mission to accelerate the pace of delivering new treatments to the patients who need them. But as groundbreaking as NCATS may be, government efforts to kickstart drug development will not be enough to bridge the divide between the basic research typically conducted by academic institutions and the large-scale clinical studies orchestrated by the pharmaceutical industry. Rather, it is the shared responsibility of all of us involved in every stage of drug development-at any phase, in the public or private sector-to contribute to filling this gap.

At the Michael J. Fox Foundation (MJFF), we've seen how this approach can pay off. We've seen how collaborations among academic and industry players are ignited when researchers share information in real time, how meeting scientists' needs for better research tools enables them to move toward breakthroughs faster and how investing in early promising therapeutic targets is crucial. Despite the inherent risks involved in these efforts, the reward could be the cure that countless patients don't have the time to wait for.

Our foundation's exclusive mission is to accelerate the development of a cure for Parkinson's disease, and over $90 \%$ of our funding is allocated to the translational and clinical research closest or most essential to patient relevance. Although we've funded only a total of about $\$ 300$ million in research to date-chump change in the context of the therapeutic development ecosystem-we believe we're on to something big. Owing in no small part to our strategic investments and untraditional collaborations with industry partners, we've seen seismic shifts in the Parkinson's therapeutic development landscape over the past decade, with major progress toward improved symptomatic and disease-modifying therapies alike.

MJFF has seen firsthand how strategic investments in translation and collaboration can pay off. For example, a new partnership between Vanderbilt University and the pharma giant Bristol-Myers Squibb (BMS) leverages investments MJFF has been making in Vanderbilt since 2005. The Tennessee-based institution's Center for Neuroscience Drug Discovery, with MJFF's funding, has been working to develop a new glutamate-based class of symptomatic treatment for Parkinson's disease. Following proof of concept in rodent models, this work has now ripened to readiness for major follow-on funding from BMS. It's a win for our foundation, Vanderbilt, BMS and, most importantly, patients with Parkinson's.

Key policymakers on Capitol Hill want to learn more about how academia, government, patient organizations and industry are working together to help speed the new treatments patients need. In September, I was invited to Washington, DC, to take part in the announcement of a new report by the US Presidential Council of Advisors on Science and Technology (PCAST) on the need for widespread, public-private collaboration to propel innovation in drug discovery and development and to be part of a panel discussion on implementing PCAST's recommendations. The week before that, ten of the world's largest biopharmaceutical companies announced the formation of TransCelerate BioPharma, a nonprofit organization that means to take a collaborative, precompetitive approach to accelerate the development of new drugs. I'm energized by the prospect of greater resources and a new framework for collaborations focused on problem-solving in the translational space, especially when today's scientists have so many promising new avenues for investigation, new targets for therapies and rapidly advancing technologies-crucial tools for progress that the field didn't know about, or that didn't exist, ten years ago.

The promise of collaboration goes beyond Parkinson's. Take, for example, how the progress enabled by the Alzheimer's Disease Neuroimaging Initiative (ADNI), a collaborative effort among multiple groups including government, drug companies, universities and nonprofits, has galvanized drug development for that disease in relatively few years Alzheimer's drugmakers have taken the proactive step of pooling data from those trials in an attempt at problemsolving and increasing the odds of better future results. Our own Parkinson's Progression Markers Initiative, supported by 13 pharmaceutical funding partners, has benefited from having ADNI as a scientific precedent and a successful model of collaboration.

Patients, for their part, are stepping up in the tens of thousands to collaborate in the scientific process, and they help define the most crucial needs for drugmakers' attention. For instance, MJFF's Fox Trial Finder, an online tool that combines matches volunteers to trials on the basis of several factors (including geography, treatment history and individual preference) and allows for anonymous two-way messaging between volunteers and clinical trial teams, has exploded since its launch by our foundation in April 2012. More than 12,000 registrants have signed up to date, and we're well on our way to the kind of volunteer army that can help make the most expensive phases of research more efficient and affordable for trial sponsors. It's a strong start, but more volunteers are needed, and greater resources are required to educate patients and their loved ones about the vital part that only they can play to keep research moving forward.

It is incumbent on us all to conceive new models that buck oldschool competition and secrecy, to share data early and often and to advocate locally and nationally for the attention, creativity and dollars that will keep science moving forward. Together, we can make cures inevitable. The choice is ours.

Todd B. Sherer is the chief executive officer of The Michael J. Fox Foundation for Parkinson's Research in New York. 